\title{
Temperature dependence of pure CsI: scintillation light yield and decay time
}

\author{
C. Amsler ${ }^{a}$, D. Grögler ${ }^{a}$, W. Joffrain ${ }^{c, 1}$, D. Lindelöf ${ }^{a}$, \\ M. Marchesotti ${ }^{\mathrm{d}, 2}$ P. Niederberger ${ }^{\mathrm{a}}, \mathrm{H}$. Pruys ${ }^{\mathrm{a}}$, \\ C. Regenfus ${ }^{\mathrm{a}}$,P. Riedler ${ }^{\mathrm{a}}$, A. Rotondi ${ }^{\mathrm{b}}$ \\ ${ }^{a}$ Physik-Institut, Zürich University, Winterthurerstrasse 190, CH-8057 Zürich, \\ Switzerland \\ ${ }^{\mathrm{b}}$ Dipartimento di Fisica Nucleare e Teorica, Università di Pavia and INFN \\ Sezione di Pavia, Pavia, Italy \\ ${ }^{\mathrm{c}}$ Dipartimento di Fisica, Università di Genova and INFN Sezione di Genova, \\ Genova, Italy \\ dPS Division, CERN, Geneva, Switzerland
}

\begin{abstract}
The temperature dependence of the light emission for pure CsI crystals has been measured with photomultipliers, and photodiodes with wavelength shifters from 80$300 \mathrm{~K}$. The light yield at $80 \mathrm{~K}$ is $N_{\gamma}=50,000 \pm 5,000$ photons per $\mathrm{MeV}$. This number was deduced from the number of electron-hole pairs produced in the photodiode, $N_{e h}=39,600 \pm 1,200$. The light yield at room temperature is lower by a factor of $15.8 \pm 1.0$, giving 3,200 \pm 400 photons per MeV. Decay times were measured with a photomultiplier. At room temperature two fast decay components were observed with decay times of $6 \pm 1 \mathrm{~ns}$ and $28 \pm 2 \mathrm{~ns}$. Below $180 \mathrm{~K}$ only one component is observed and at $80 \mathrm{~K}$ the decay time is $1015 \pm 17 \mathrm{~ns}$.
\end{abstract}

Email address: christian.regenfus@cern.ch (C. Regenfus).

URL: http://afuz01.cern.ch/zuerich.html (C. Amsler).

1 Present address: Physics Department and Center for Materials Science and Engineering, Massachusetts Institute of Technology, Cambridge, MA 02139, USA

2 on leave of absence from Dipartimento di Fisica Nucleare e Teorica, Università di Pavia, Pavia, Italy

Preprint submitted to Elsevier Science

27 March 2001 


\section{Introduction}

The ATHENA (AnTiHydrogEN Apparatus [1]) experiment will produce and study antihydrogen atoms at low energies. The formation of antihydrogen atoms is studied in a Penning trap, where antiprotons and positrons are put in close contact. After the recombination of an antiproton and a positron the formed neutral antihydrogen atom is no longer confined in the trap region and annihilates on the trap wall. The production rate of antihydrogen atoms is monitored by a large solid angle and high granularity charged particle and $\gamma$-ray detector. This detector detects simultanously the pions from antiprotonnucleon annihilation and the $511 \mathrm{keV} \gamma$-rays from positron-electron annihilation. The pions are detected in silicon microstrip detectors and the $\gamma$-rays in pure CsI crystals. Since the recombination trap works at very low temperature $(\simeq 1 \mathrm{~K})$ the surrounding annihilation detector cannot operate at room temperature. With good insulation the detector can work in a temperature range of $80-120 \mathrm{~K}$. In this paper we describe the scintillation properties of pure CsI crystals in the temperature range 80 - $300 \mathrm{~K}$. The properties of the silicon microstrip detector modules and the frontend readout electronics are described in another paper [2].

Pure CsI is used at room temperature as a fast scintillator (decay time $\simeq 10$ $\mathrm{ns}$ ) in electromagnetic calorimeters at high counting rates [3,4]. However, the light yield at room temperature is only about $8 \%$ relative to $\mathrm{NaI}(\mathrm{Tl})$. Earlier investigations $[5,6]$ show a dramatic change of the scintillation properties of pure CsI at low temperatures. By going from room temperature to the temperature of liquid nitrogen $(77 \mathrm{~K})$ the light yield increases by about a factor of 10 , but the decay time increases to about $1 \mu \mathrm{s}$. Since the published results are not very precise and even contradictory, a new investigation was necessary. In this paper we describe those properties of pure CsI which are important for a $511 \mathrm{keV} \gamma$-ray detector at low temperatures. The experimental methods for reading out the UV scintillation light of pure CsI crystals are described in section 2 . Both UV sensitive photomultipliers and silicon photodiodes with a UV to red wavelength shifter were used. The results, the temperature dependence of the light yield and decay times and the absolute light yield at $77 \mathrm{~K}$, are given in section 3 and compared with previously published results. The most important results are summarized in section 4 . 


\section{Experimental procedures}

Polished pure CsI crystals were obtained from Tbilisi State University (dimensions $\left.20 \times 20 \times 45 \mathrm{~mm}^{3}\right)$ and CRYSMATEC $\left(13.0 \times 17.1 \times 17.6 \mathrm{~mm}^{3}\right.$, the final dimensions in the ATHENA detector). For maximum light output, the crystals were wrapped with white reflecting teflon tape. The crystals were mounted in a vacuum chamber where they could be cooled down to the temperature of liquid nitrogen $(77 \mathrm{~K})$. The temperature dependent measurements were done by slowly raising the temperature from $77 \mathrm{~K}$ to $295 \mathrm{~K}$. The temperature was

monitored with a PT2000 temperature sensor.

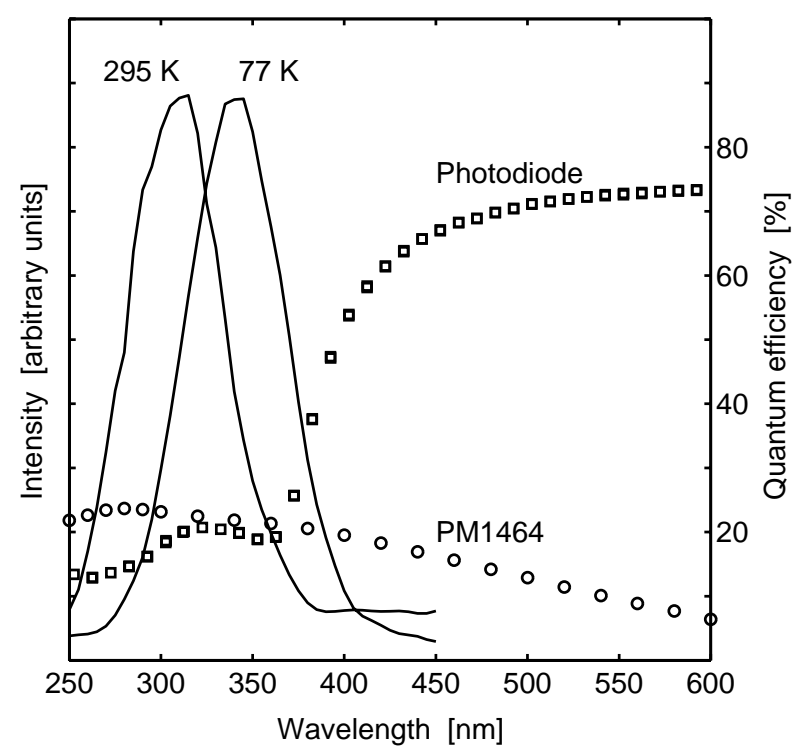

Fig. 1. Emission spectrum of pure CsI at two different temperatures (solid lines [5]) and quantum efficiencies of the photomultiplier (data from Hamamatsu [7]) and the silicon photodiode.

The wavelength of the scintillation light of pure CsI is in the UV region with a maximum emission at $310 \mathrm{~nm}$ at room temperature and $340 \mathrm{~nm}$ at $77 \mathrm{~K}$ (fig. 1). To control the systematic errors we used two different readout methods. First, we used a UV sensitive $3 / 4$ inch diameter photomultiplier (PM) from Hamamatsu (R1464) with a quantum efficiency $Q=22.8 \%$ at $310 \mathrm{~nm}$ and $Q=21.9 \%$ at $340 \mathrm{~nm}$ (fig. 1). The PM was kept at room temperature and coupled to the crystal with a quartz rod without optical grease, resulting in a low and unknown light collection efficiency. For the measurement of the light yield as function of the temperature (see section 3.1) the anode output of the PM was connected with a scintillation preamplifier (ORTEC 113) and a spectroscopic amplifier (CANBERRA 1413, $6 \mu$ s shaping time). The decay times (section 3.2) were determined by measuring directly the output pulses from the anode with a $400 \mathrm{MHz}$ digital oscilloscope. 


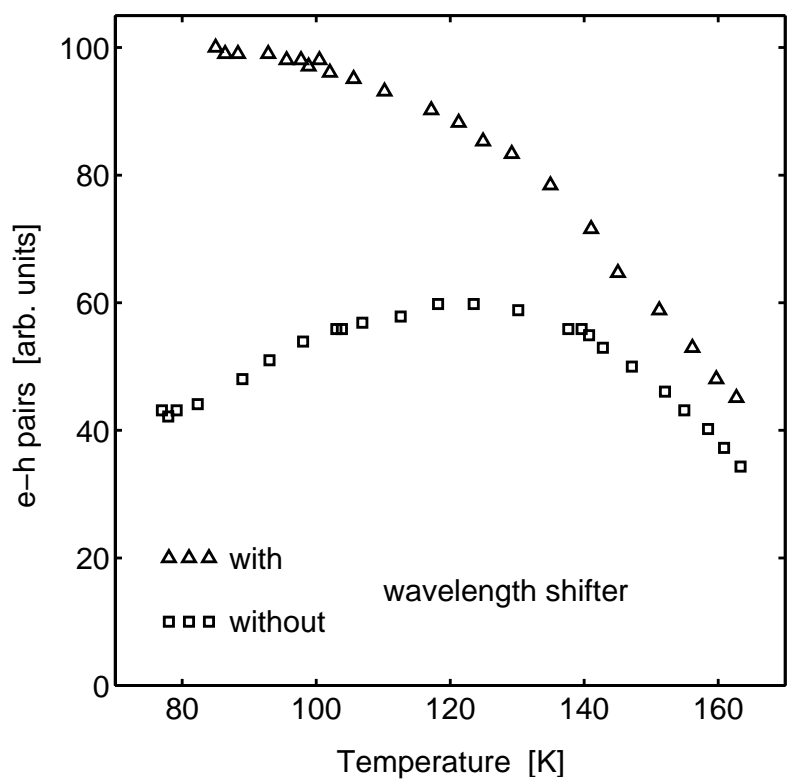

Fig. 2. Relative yield of electron-hole pairs measured with a pure CsI crystal and a photodiode, with and without a UV to red wavelength shifter. The yield is normalized to the maximum of the measurement with the wavelength shifter.

As a second readout method, we used a $1.6 \mathrm{~cm}^{2}$ silicon photodiode from SINTEF (thickness $380 \pm 15 \mu \mathrm{m}$, operating voltage $30-70 \mathrm{~V}$ ). The quantum efficiency $Q$ of a photodiode is strongly affected by the wavelength-dependent reflectivity of silicon $[8,9]$. The measured quantum efficiency (fig. 1) shows that this effect is very critical in the UV region below $400 \mathrm{~nm}$. When the diode is coupled to a CsI crystal the effective quantum efficiency is higher, since the photons reflected from the diode surface are also reflected from the crystal surfaces and have a second chance to enter the photodiode. The influence of the geometry on the effective quantum efficiency has been studied by Holl et al. [10] and effective quantum efficiencies over $90 \%$ could be obtained for red light. To get a high and wavelength independent quantum efficiency we used a UV to red wavelength shifter. The readout face of the crystal was coupled to the photodiode with silicon grease. The remaining faces were painted with red dye (MODEL MASTER Fluorescent Red <FS28915>, ITALERI, 40012 Calderara, Italy) and wrapped with white reflecting teflon tape. The signal of the photodiode was amplified in a preamplifier (CANBERRA 2003 BT) at room temperature outside the cryostat, and further processed in a spectroscopic amplifier (TENNELEC TC $205 \mathrm{~A}$ with $8 \mu$ s shaping time). The large improvement due to the wavelength shifter (more than a factor of 2 at $85 \mathrm{~K}$ ) is shown in fig. 2 . 


\section{Results}

\subsection{Light yield}

Light yield measurements were made with the photomultipliers and photodiodes using the $511 \mathrm{keV}$ and $1275 \mathrm{keV} \gamma$-rays from a ${ }^{22} \mathrm{Na}$ source. As an example, $\gamma$-ray spectra taken with a pure CsI crystal at two different temperatures are given in fig. 3. The positions of the $511 \mathrm{keV}$ and $1275 \mathrm{keV}$ photopeaks clearly depend strongly on the temperature. The peak positions are proportional to number of photoelectrons or electron-hole pairs produced in the photomultiplier or photodiode. They are proportional to the number of scintillation photons produced in the crystal by the absorption of the $\gamma$-ray. The accurate positions of the peaks were determined by fitting gaussians with quadratic background in the peak region.

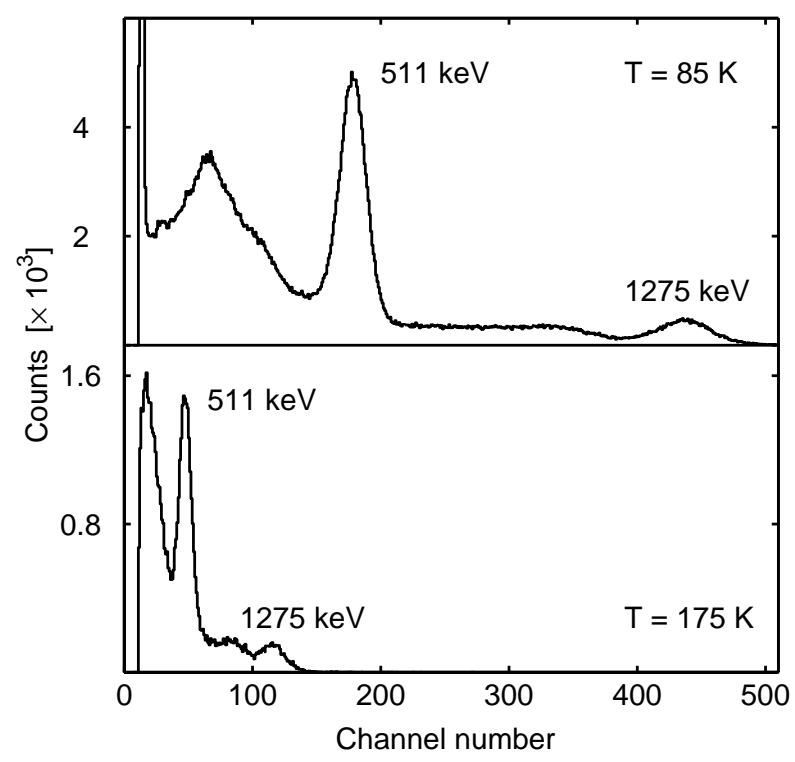

Fig. 3. ${ }^{22} \mathrm{Na}$ spectra taken with a pure CsI crystal coupled to a photomultiplier (Hamamatsu R1464) at two different temperatures.

The relative light yield (normalized to the maximun yield) as a function of temperature in the range $80 \mathrm{~K}$ to $300 \mathrm{~K}$ is given in fig. 4. The measurements with the photomultiplier and the photodiode are in good agreement. The strongest temperature dependence occurs around $150 \mathrm{~K}$ (a temperature difference of $1 \mathrm{~K}$ leads to a $1 \%$ shift in the peak postion). For maximum light yield and thus best resolution, the CsI crystals in the ATHENA detector should be kept at a constant temperature below $100 \mathrm{~K}$. Operating the crystals in the region around $150 \mathrm{~K}$ is only acceptable if the temperature can be kept constant within a few degrees. 


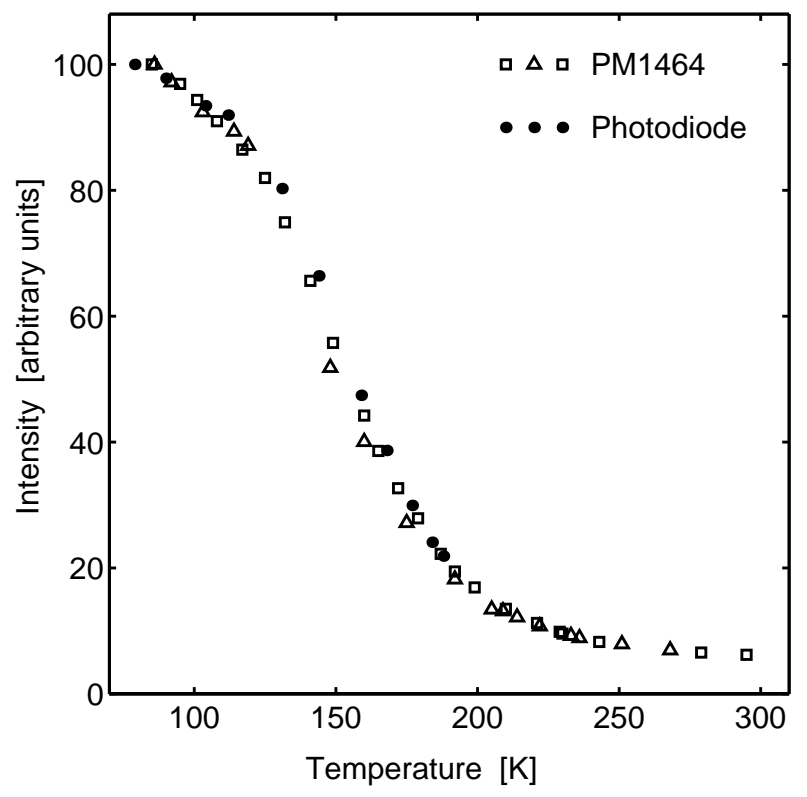

Fig. 4. Light yield of pure CsI crystals as a function temperature. Both measurements, one with a photomultiplier (Hamamatsu R1464), and the other with a photodiode (SINTEF) and wavelength shifter, are normalized independently to the maximum.

With photomultipliers and photodiodes contradictory results were reported by Woody et al. [5]. With a photodiode they found an increase in light yield by 14.6 when cooling down from $295 \mathrm{~K}$ to $77 \mathrm{~K}$. This agrees with our result

$$
\frac{N_{\gamma}(77 \mathrm{~K})}{N_{\gamma}(295 \mathrm{~K})}=15.8 \pm 1.0 .
$$

However, Woody et al. [5] measured with a photomultiplier an increase in light yield by only a factor of 6 . The reason for this discrepancy is not known.

The absolute light yield was determined by calibrating the photodiode with a ${ }^{109} \mathrm{Cd}$ source (fig. 5). A $88 \mathrm{keV}$ photon produces in silicon on average 24,400 electron-hole pairs. The center of the $88 \mathrm{keV}$ photopeak is in channel $109 \pm 2$. With the same setup, the total absorption of $662 \mathrm{keV} \gamma$-rays (from a ${ }^{137} \mathrm{Cs}$ source) in the CsI crystal leads to a peak in channel $116.9 \pm 0.2$. Combining these numbers we get the number of electron-hole pairs in the silicon diode for the absorption of a $\gamma$ in the CsI crystal:

$$
N_{e h}(77 \mathrm{~K})=(39,600 \pm 1,200) / \mathrm{MeV}
$$

The high number, reported here for the first time for a pure CsI crystal at the temperature of liquid nitrogen, is only $17 \%$ lower than the number reported by Valentine et al. [8] for thallium-activated CsI at room temperature $N_{e h}[\mathrm{CsI}(\mathrm{Tl}), 295 \mathrm{~K}]=47,800 \pm 1,200$. The authors determined the wavelength-averaged quantum efficiency $\bar{Q}=91 \%$ for a silicon photodiode 


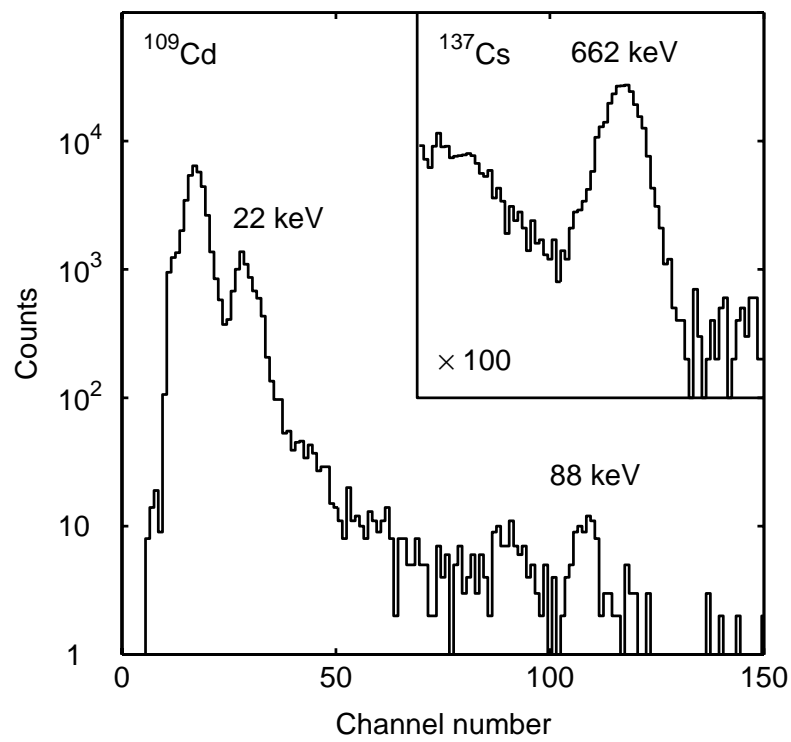

Fig. 5. Calibration of the photodiode with X-rays from a ${ }^{109} \mathrm{Cd}$ source at $77 \mathrm{~K}$. The peaks produced by the photoeffect of the $22 \mathrm{keV}$ and $88 \mathrm{keV}$ photons in the silicon diode are observed. The peak just above threshold is due to scintillation photons generated by the absorption of the $88 \mathrm{keV}$ X-rays in the CsI crystal. The inset shows the photopeak produced by the absorption of the $662 \mathrm{keV} \gamma$-rays from a ${ }^{137} \mathrm{Cs}$ source.

and for the scintillation light of $\mathrm{CsI}(\mathrm{Tl})$ (peak emission at $550 \mathrm{~nm}$ ). The quantum efficiency for red light $(\simeq 650 \mathrm{~nm})$ was found to be even higher, $Q_{r} \simeq 97 \%$. With Monte Carlo simulation they also estimated a light collection efficiency of $\eta=0.81 \pm 0.03$ for their scintillator-photodiode assembly. Since their geometry is very similar to ours, we assume the same efficiency. Our estimate for the number of produced scintillation photons in pure CsI is then

$$
N_{\gamma}(77 \mathrm{~K})=\frac{N_{e h}(77 \mathrm{~K})}{\eta Q_{r}}=(50,000 \pm 5,000) / \mathrm{MeV}
$$

This large yield is only $20 \%$ lower than the $63,000 \pm 3,000$ photons per $\mathrm{MeV}$ obtained for $\mathrm{CsI}(\mathrm{Tl})$ at $295 \mathrm{~K}[8,9]$. Such a yield is also important to get a good resolution. By using a preamplifier directly coupled to the photodiode at $77 \mathrm{~K}$ (the final setup in the ATHENA detector [2]) we could reduce the electronic noise and get a energy resolution of $\Delta E(\mathrm{FWHM}) / E=8.3 \%$ for $511 \mathrm{keV} \gamma$-rays (fig. 6).

From the absolute number of photons $N_{\gamma}(77 \mathrm{~K})$ measured at the temperature of liquid nitrogen and the measured ratio $N_{\gamma}(77 \mathrm{~K}) / N_{\gamma}(295 \mathrm{~K})$ we get the number of photons at room temperature

$$
N_{\gamma}(295 \mathrm{~K})=(3,200 \pm 400) / \mathrm{MeV}
$$

which is $(5.1 \pm 0.7) \%$ of the $\mathrm{CsI}(\mathrm{Tl})$ yield, in agreement with the $6 \%$ obtained by Woody et al. [5]. Similar results have been reported for the ratio of pure 


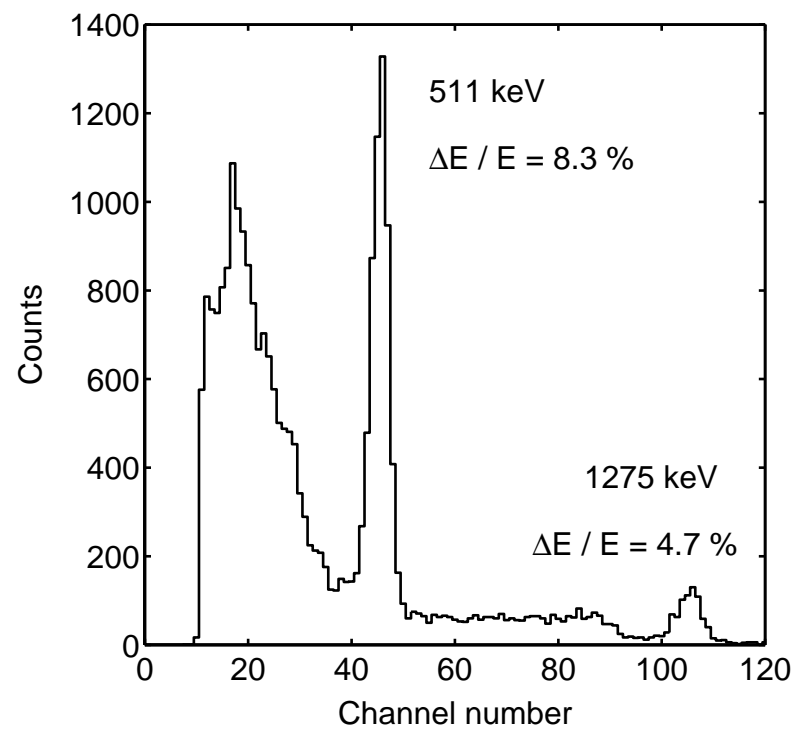

Fig. 6. ${ }^{22} \mathrm{Na}$ spectrum taken with a pure CsI crystal sprayed with the red dye and coupled to a photodiode and preamplifier, at the temperature of liquid nitrogen.

CsI to NaI(Tl) light yields, $0.05-0.08$ [3] and 0.06 [13]. These results are in disagreement with the $16,800 \pm 870$ photons per $\mathrm{MeV}$ at room temperature obtained by Holl et al. [10]. The discrepancy is probably due to impurities in their crystals. The scintillation light yield of "pure" CsI crystals at room temperature depends very strongly on the quality of the crystal. The properties of poor crystals with intrinsic vacancies and/or impurities, resulting in a slow $(\simeq 1 \mu \mathrm{s})$ emission component, has been discussed in the literature $[13,14]$.

\subsection{Decay times}

Decay times of the scintillation light for pure CsI were measured with the photomultiplier in the range between room temperature $(295 \mathrm{~K})$ and $85 \mathrm{~K}$. Two time components were observed at high temperatures $(192-295 \mathrm{~K})$ and the measured light intensity could be described by the sum of two exponential decays

$$
I(t)=\frac{I_{1}}{\tau_{1}} e^{-t / \tau_{1}}+\frac{I_{2}}{\tau_{2}} e^{-t / \tau_{2}} .
$$

This is illustrated at $192 \mathrm{~K}$ in fig. 7 . Our results at room temperature

$$
\tau_{1}=6 \pm 1 \mathrm{~ns}, \quad \tau_{2}=28 \pm 2 \mathrm{~ns}, \quad \frac{I_{1}}{I_{1}+I_{2}}=0.21 \pm 0.02
$$

are in good agreement with the results of Woody et al. [5], $\tau_{1} \simeq 7 \mathrm{~ns}, \tau_{2} \simeq 29$ ns. The decay times reported by Kubota et al. [3] are somewhat higher, $\tau_{1}=10$ $\mathrm{ns}, \tau_{2}=36 \mathrm{~ns}$. In addition to these two components, $\tau_{1}=5-9 \mathrm{~ns}, \tau_{2}=10-25$ ns, Belsky et al. [11] also observed a very fast component with 0.7-2 ns decay 


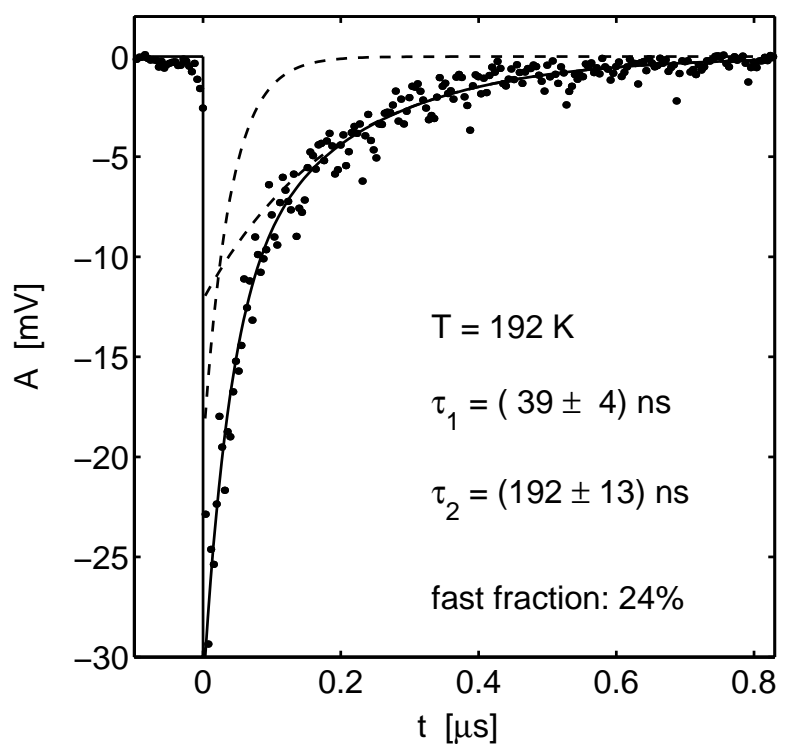

Fig. 7. Light emission curve for a pure CsI crystal at $192 \mathrm{~K}$, measured with a photomultiplier. The main component ( $76 \%$ of the total light yield) with a decay time of $192 \mathrm{~ns}$ and the fast component $(24 \%)$ with a decay time of $39 \mathrm{~ns}$ are clearly seen (dashed curves).

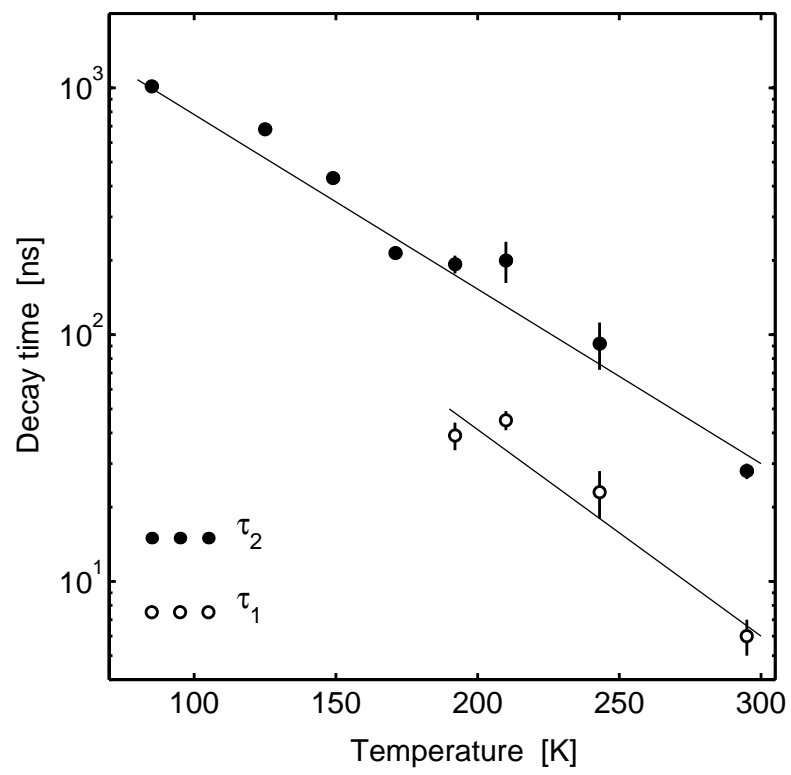

Fig. 8. Decay times of the light emission for a pure CsI crystal at different temperatures. The eyeball straight line fits show the general temperature dependence.

time, which was also observed by other investigators $(2.1 \pm 0.2 \mathrm{~ns}$ in ref. [4], $1.8 \mathrm{~ns}$ in ref. [12]).

Our measured decay times, in the range 190-295 K (fig. 8), are generally in agreement with previous results [5,6]. Below $192 \mathrm{~K}$, the light emission curve could be described by one exponential decay only (fig. 9) and the measured 


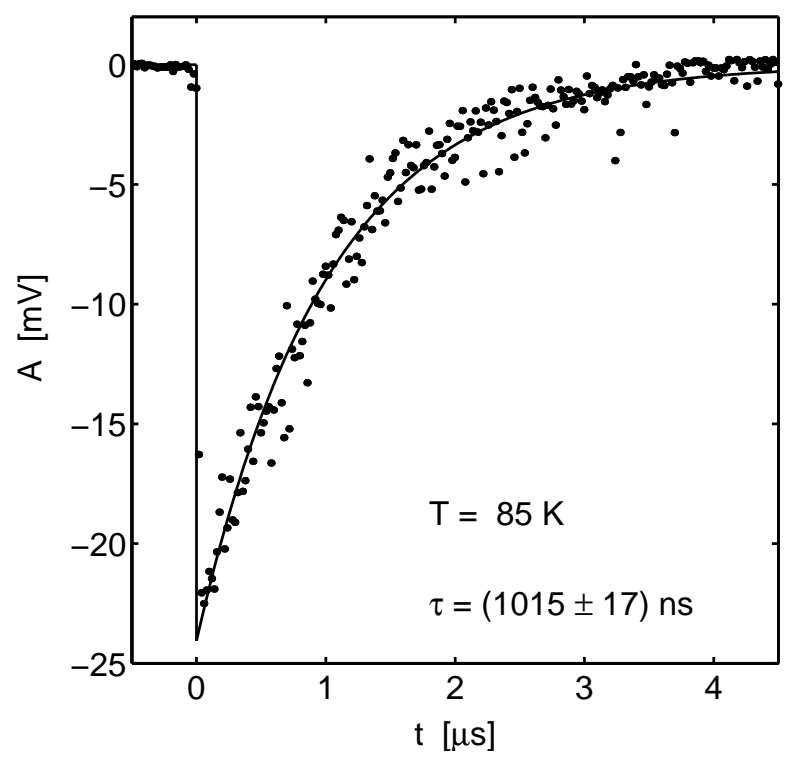

Fig. 9. Light emission curve for a pure CsI crystal at $85 \mathrm{~K}$, measured with a photomultiplier. Only one slow component with a decay time of about $1 \mu \mathrm{s}$ is observed.

decay times are in agreement with the results of Nishimura et al. [6]. However, Woody et al. [5] still report two components below $190 \mathrm{~K}$. At $105 \mathrm{~K}$ their decay times are relatively short, $\tau_{1} \simeq 180 \mathrm{~ns}$ and $\tau_{2} \simeq 320 \mathrm{~ns}$, in clear disagreement with the long decay time of $1 \mu$ s observed in the present work.

\section{Summary}

Pure CsI crystals can be used as fast scintillators at room temperature. The decay curve shows two components with decay times of $6 \pm 1 \mathrm{~ns}$ and $28 \pm 2 \mathrm{~ns}$. A third, ultrafast component $(\simeq 2 \mathrm{~ns})$ has been reported in the literature. However, the light yield of 3,200 \pm 400 photons per MeV is only $5 \%$ compared to thallium-activated CsI. These quantitative results are in agreement with the more qualitative results reported in the literature.

With a silicon photodiode and a UV to red wavelength shifter, we have shown that pure CsI is a good scintillator at the temperature of liquid nitrogen $(77 \mathrm{~K})$ with properties similar to $\mathrm{CsI}(\mathrm{Tl})$ at room temperature. The number of measured electron-hole pairs is $39,600 \pm 1,200$ per $\mathrm{MeV}$, leading to a light yield of 50,000 $\pm 5,000$ photons per $\mathrm{MeV}$. The energy resolution measured with a preamplifier also at $77 \mathrm{~K}$ is $\Delta E(\mathrm{FWHM}) / E=8.3 \%$ for $E=511 \mathrm{keV}$. The decay curve measured with a photomultiplier shows only one component with a decay time of $1015 \pm 17 \mathrm{~ns}$. Pure CsI crystals operating at 80-120 K are used in the ATHENA antihydrogen detector, which will come into full operation in summer 2001. 


\section{Acknowledgments}

We thank D. Mzavia (University of Tbilisi) for supplying us with good quality pure CsI crystals, B. Schmid, E. Hermes (University of Zürich) and S. Bricola, C. Marciano (University of Pavia) for their help in the construction of the experimental setup. D. Clement and J.-F. Loude (University of Lausanne) are gratefully acknowledged for the loan of their equipment and their help in measuring the quantum efficiency of the photodiode. We are also grateful to A. N. Belsky, P. Lecoq and E. Auffray (Crystal Clear collaboration, CERN)

for discussions related to the scintillation mechanism of pure CsI crystals.

\section{References}

[1] ATHENA proposal, CERN SPSLC 96-47, see http://www.cern.ch/athena/

[2] R. A. Brunner, Diplomarbeit, Universität Zürich (2000) unpublished;

C. Amsler et al., to be published

[3] S. Kubota et al., Nucl. Instr. Meth. A268 (1988) 257.

[4] P. Schotanus et al., IEEE Trans. Nucl. Sci. 37 (1990) 177.

[5] C. L. Woody et al., IEEE Trans. Nucl. Sci. 37 (1990) 492.

[6] H. Nishimura et al., Phys. Rev. B51 (1995) 2167.

[7] Hamamatsu, Photomultiplier Tubes (1985)

[8] J. D. Valentine et al., IEEE Trans. Nucl. Sci. 40 (1993) 1267.

[9] M. Moszynski et al., IEEE Trans. Nucl. Sci. 44 (1997) 1052.

[10] I. Holl, E. Lorenz and G. Mageras, IEEE Trans. Nucl. Sci. 35 (1988) 105.

[11] A. N. Belsky et al., Rev. Sci. Instr. 63 (1992) 806.

[12] S. Keszthelyilandori et al., Nucl. Instr. Meth. 303 (1991) 374.

[13] B. K. Utts and S. E. Spagno, IEEE Trans. Nucl. Sci. 37 (1990) 134

[14] M. M. Hamada et al., Nucl. Instr. Meth. A365 (1995) 98 